\title{
Selective Laser Sintering Method Using Titanium Powder Sheet Toward Fabrication of Porous Bone Substitutes*
}

\author{
Terutake HAYASHI**, Katsuhiro MAEKAWA**, \\ Masashi TAMURA*** and Kenichi HANYU $^{* * *}$
}

\begin{abstract}
The present paper investigates the laser sintering of titanium sheets toward the fabrication of porous artificial bones. The novelty lies in the use of a titanium powder sheet mixed with an organic binder and the application of selective laser sintering to the fabrication of a laminated porous structure. Alternating irradiation of Nd:YAG pulses with short scanning paths results in the suppression of distortion of the sintered part as well as enhanced mechanical properties. Under the appropriate conditions identified in the experiment, a bending strength of $63 \mathrm{MPa}$ and a Young's modulus of $1.5 \mathrm{GPa}$ are attained when the load is applied parallel to the lamination direction, whereas load vertical to the lamination direction yields $79 \mathrm{MPa}$ and $1.8 \mathrm{GPa}$, respectively. The size of pores varies from 200 to $300 \mu \mathrm{m}$, and the porosity is approximately $65 \%$. These values, other than Young's modulus, are almost equivalent to those of human bones.
\end{abstract}

Key Words: Laminated Construction, Biomaterials, Powder Metallurgy, Selective Laser Melting, Titanium, Artificial Bone, Porous Structure, Rapid Prototyping, Layer Manufacturing

\section{Introduction}

Porous metal materials have various applications such as in light weight structures and filters, since they possess sophisticated properties, including large surface area, low density and high thermal insulation, in comparison with conventional materials. Porous titanium in particular draws attention as a structural material for bone substitutes and dental implants, because of its intrinsic light weight and the biocompatibility.

Precise control over the scaffold material, porosity and internal pore architecture is essential for tissue engineering. By adding osteogenic growth factors to synthetic bone substitutes, the amount of new bone fabrication could be increased and the clinical outcome improved $^{(1)}$. After undergoing specific chemical and thermal treatments, porous bioactive titanium induces bone formation without the need for additional osteogenic cells or os-

${ }^{*}$ Received 17th May, 2005 (No. 05-4133)

** Division of Laser Micro Fabrication, The Research Center for Superplasticity, Faculty of Engineering, Ibaraki University, 4-12-1 Nakanarusawa, Hitachi, Ibaraki 3168511, Japan. E-mail: mae@mech.ibaraki.ac.jp

*** Graduage School of Science and Engineering, Ibaraki University teoinductive agents ${ }^{(2)}$.

Recently, mouldless manufacturing techniques, known as solid free-form fabrication $(\mathrm{SFF})^{(3)}$, or rapid prototyping have been successfully used to fabricate complex scaffolds ${ }^{(4)}$. Titanium cranioplasty plates are manufactured using 3-D computer modeling from CT scan data, computer-driven milling and selective laser sintering ${ }^{(5),(6)}$. By coupling SFF with conventional sponge scaffold fabrication procedures, methods for casting polymer-ceramic scaffolds with the designed porous internal architectures have been developed ${ }^{(7)}$.

In the present paper, a process of selective laser sintering using a titanium powder sheet is investigated for the fabrication of porous bone substitutes. The novelty lies in the use of a sheet formed by mixing titanium powder and organic agents. The sheet is sintered layer-by-layer under argon atmosphere using a pulsed Nd:YAG laser beam. Operating parameters, such as laser irradiation and scanning paths, are extensively examined to suppress distortion, to increase strength and to adjust the pore size of the sintered part.

\section{Experimental Procedure}

Figure 1 illustrates a process of fabricating titanium porous structures. This process is based on layered man- 
ufacturing. A thin sheet made from titanium powder is arranged beforehand, and then sintered using a scanning laser beam. The iterations of sheet lamination and laser sintering lead to the formation of three-dimensional structures. Detailed procedures for fabricating an artificial bone from a porous titanium sheet are described below.

First, to determine the paths for laser scanning, the bone shape is measured, and then the data is transferred to the 3-D CAD system. The software generates form data as a series of cross sections in the STL (stereo lithography) format. The form data is then converted into NC (numerical control) data that define the paths for laser scanning inside the cross-sectional contours of the bone shape. Following the NC data, a porous titanium sheet is sintered with a pulsed Nd:YAG laser in an argon gas atmosphere. The use of argon prevents rapid oxidization of the melting titanium. The titanium powder included in the sheet is melted and solidified in a selected cross-sectional area of the artificial bone. After the sintering procedure, a fresh titanium sheet is placed on top of the preceding sheet, pressurized further, and a laser beam is selectively irradiated again.

A thin porous sheet is formed as follows using titanium powder with organic agents. First, gas-atomized spherical titanium powder with $82.95 \mu \mathrm{m}$ mean particle diameter, an organic binder and a solvent are mixed. The mixed slurry is poured onto a plastic sheet, covered with another plastic sheet and leveled to a specific thickness by rolling. Following that, a drying process is needed to evaporate the solvent. The mixture ratio of pure titanium and the binder is determined by considering formability, sinterability and tractability of the sheet; an appropriate combination is $6 \mathrm{vol} \%$ titanium and $94 \mathrm{vol} \%$ organic binder and solvent. The thickness of the tape is $220 \pm 30 \mu \mathrm{m}$, or the largest variation in thickness becomes about $15 \%$.

The laser parameters for sintering a titanium sheet are examined in order to fabricate porous structures with high porosity and strength, as well as uniformly dispersed pores. Figure 2 depicts the adjustable parameters of the laser processing machine, including the average power during irradiation, pulse width, peak power and frequency. A pulsed Nd:YAG laser beam with a wavelength

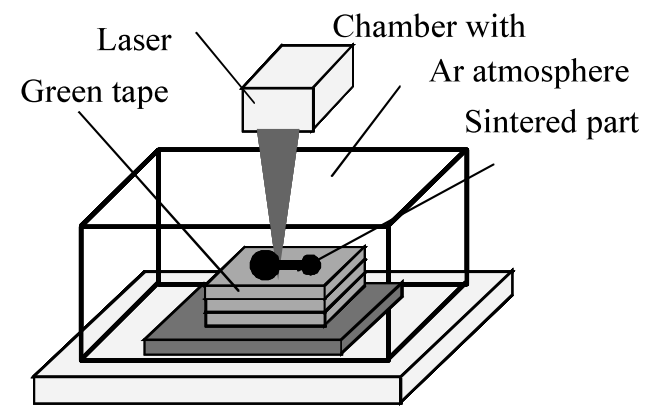

Fig. 1 Schematic of sheet lamination with laser scanning of $1064 \mathrm{~nm}$ was used with a beam spot size of $350 \mu \mathrm{m}$. Table 1 lists the two types of sintering conditions, $\mathrm{P} 1$ and $\mathrm{P} 2$, where the pulse frequency is kept constant at $80 \mathrm{~Hz}$. The condition of $\mathrm{P} 1$ corresponds to laser cutting of metal, in which the power density is low and the peak power is high because of the short pulse width. In contrast, P2 is similar to the condition of seam welding, where the pulse width is long and the average power is high. Other parameters are set as follows: a scan speed of $3.3 \mathrm{~mm} / \mathrm{s}$ and scan spacing (distance between neighboring scan paths) of $0.2 \mathrm{~mm}$. Consequently, the overlap rate of scan paths is $75 \%$ in this case.

Figure 3 shows the surface morphology when the titanium sheet was sintered under the $\mathrm{P} 1$ and $\mathrm{P} 2$ conditions of (a) low average power and (b) high average power. In the case of P1, the melted area of titanium particles is limited on their surfaces, so that the spherical shape remains on the scanned area. In the case of P2, where the average power is 3.4 times higher than P1, the titanium powder is completely melted, so that a smooth surface with little unevenness is obtained. Accordingly, the laser beam with a long pulse width and a low peak power effectively melts titanium particles in the sheet, which seems to yield a single layer with high density and high strength. However, the condition of P2 is not suitable for the fabrication of porous structures due to the degradation of the original powder shape and the short distance between the neighboring particles.

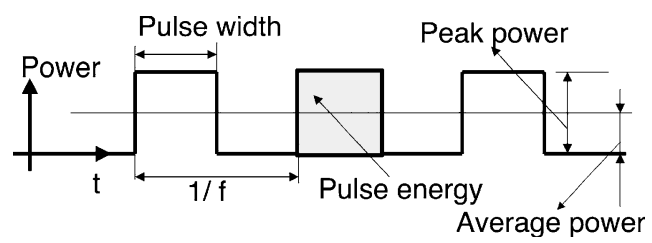

Fig. 2 Schematic diagram of pulse laser parameters

Table 1 Parameters of laser sintering

\begin{tabular}{c|c|c|c|c}
\hline & $\begin{array}{c}\text { Pulse } \\
\text { width } \\
(\mathrm{ms})\end{array}$ & $\begin{array}{c}\text { Power } \\
\text { density } \\
\left(\mathrm{W} / \mathrm{mm}^{2}\right)\end{array}$ & $\begin{array}{c}\text { Pulse } \\
\text { energy } \\
(\mathrm{J})\end{array}$ & $\begin{array}{c}\text { Peak } \\
\text { power } \\
(\mathrm{W})\end{array}$ \\
\hline P1 & 0.45 & 3.3 & 0.041 & 91.0 \\
\hline P2 & 4.5 & 11.1 & 0.14 & 31.0 \\
\hline
\end{tabular}

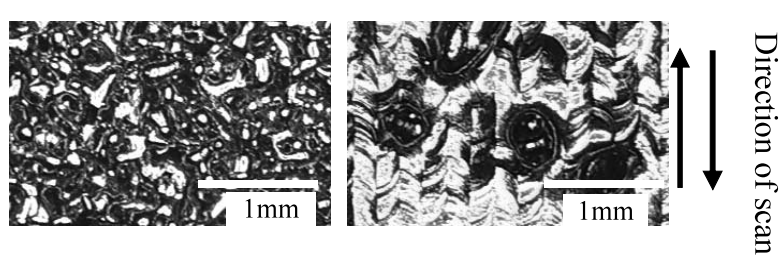

(a) Low average power (b) High average power

Fig. 3 Images of the melted surface textures 
In the case of $\mathrm{P} 1$, the strength between the sintered sheets decreases because of a shortage of the melted titanium caused by low average power. The insufficient melting takes place particularly on the opposite side of the sintered surface, causing interfacial debonding between neighboring layers. The separation is unfavorable for fabricating three-dimensional objects with pore networks. To avoid this phenomenon, it is necessary to increase the penetration depth of the melted titanium into the sheet, as well as to improve the strength of the single sintered layer.

This can be achieved by laser irradiation at a high peak power and long pulse width as compared with the case of P1. In this case, it is possible to strongly bond the neighboring titanium particles while maintaining the surface structure within the titanium sheet. We investigated some laser parameters in order to find appropriate ones, as described in section 3.3.

\section{Experimental Results}

\subsection{Bending strength}

Figure 4 shows the specimen size $\left(3 \times 30 \mathrm{~mm}^{2}\right)$ and two ways of scanning the laser beam. The specimen consists of 10 laminated sheets. The laser sintering condition is the same as P1 shown in Table 1. A three-point bending test was carried out in two ways, as shown in Fig. 5: (a) one is to apply load in the direction parallel to the lamination, and (b) the other, vertical to the lamination. The results were $24.6 \mathrm{MPa}$ and $14.6 \mathrm{MPa}$, respectively. The porosity of the specimen was $67 \%$.

A bending strength of $30-180 \mathrm{MPa}$ is necessary for human bones ${ }^{(8)}$. The results are much lower than the required strength. In order to improve the strength of the specimens by the proposed method, it is important to examine the fracture mechanism of the specimen. It is considered that the bending strength arises from the bonding strength of neighboring layers when the load is applied parallel to the lamination direction. This load can cause

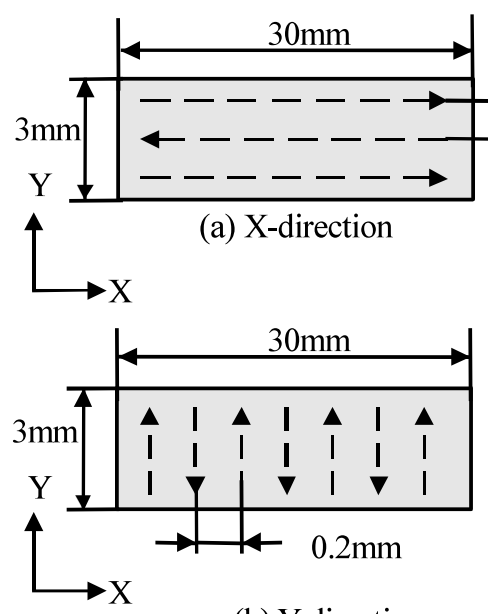

(b) Y-direction

Fig. 4 Schematic of laser scanning path the separation of neighboring layers. The bending strength is also affected by the compressive and tensile strength of the sintered layer when the load is applied vertical to the lamination direction. The load compresses the sintered layer on the upper side and pulls at the back. Therefore, it is necessary to increase both the bonding strength of neighboring layers and the compressive strength of the sintered layer. This point will be discussed further in section 3.4.

\subsection{Distortion}

The distortion or warpage of the specimen is also a cause of reduced bonding strength between neighboring layers. The degree of warping was examined for both laser scanning paths, as shown in Fig. 4: a long scanning path in the X-direction and a short one in the Y-direction. The deformation of the sintered layer was measured at 7 points in $5 \mathrm{~mm}$ intervals, as shown in Fig. 6 (a).

Figure 6(b) shows the result. Shrinkage is increased at both ends of the specimen in the case of X-direction sintering. In this case, the length of the specimen varies from $2.9 \mathrm{~mm}$ to $3.6 \mathrm{~mm}$ on the narrow side, and the difference is $0.7 \mathrm{~mm}$. In the case of Y-direction sintering, the specimen is expanded uniformly at all points; the difference

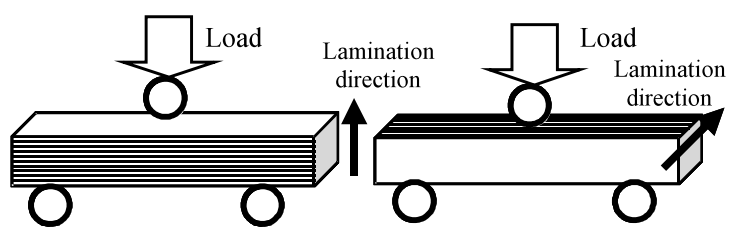

$\begin{array}{ll}\text { (a) Parallel direction } & \text { (b) Vertical direction }\end{array}$

Fig. 5 Schematic of three-point bending test

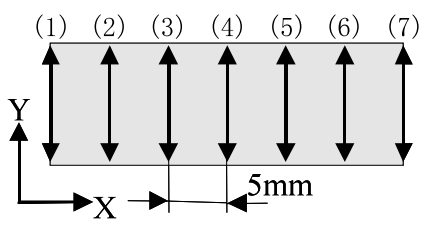

(a) Measurement positions

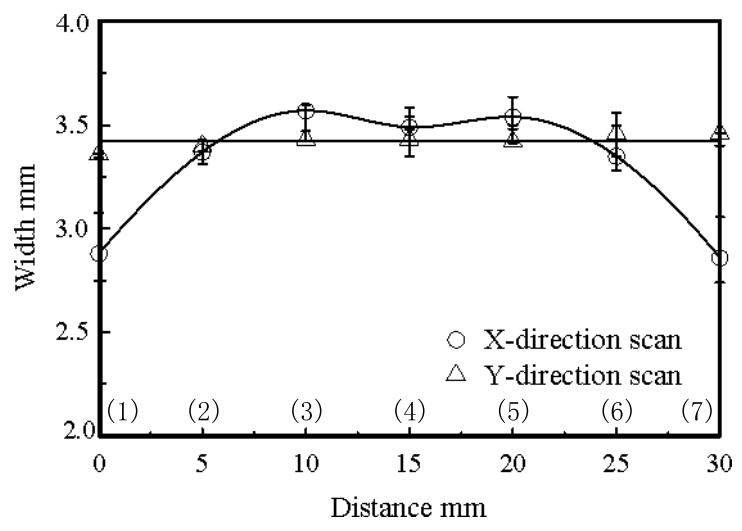

(b) Distortions in X-Y plane

Fig. 6 Distortion of two scanning paths 
in the length is only $0.1 \mathrm{~mm}$. Accordingly, the repetition of scans with short during laser sintering is effective for decreasing the unequal warpage deformation.

\subsection{Influence of laser scan spacing on tensile strength and porosity}

The influence of scan spacing, or the distance between neighboring scan paths, on tensile strength was investigated. The size of the specimen and the laser scanning paths are the same as those in Fig. 4(b). The laser irradiation parameters were altered to a pulse width of $2.2 \mathrm{~ms}$ and an average power of $15.1 \mathrm{~W}$, where pulse frequency of $80 \mathrm{~Hz}$ and scan speed of $3.3 \mathrm{~mm} / \mathrm{s}$ were unchanged. The scan spacing varied from $0.1 \mathrm{~mm}$ up to $0.4 \mathrm{~mm}$. Five specimens were prepared in each case.

Figure 7 shows the relationship between the tensile strength and the scan spacing. The tensile strength decreased with increasing scan spacing, whereas the porosity increased. The maximum tensile strength of $36 \mathrm{MPa}$ was obtained at a scan spacing of $0.15 \mathrm{~mm}$. In addition, the maximum Young's modulus of $1.66 \mathrm{GPa}$ was obtained at a scan spacing of $0.2 \mathrm{~mm}$. When the porosity shows a maximum value of $40 \%$ at a scan spacing of $0.1 \mathrm{~mm}$, the tensile strength is as high as $35 \mathrm{MPa}$. However, Young's modulus was $1.07 \mathrm{GPa}$, which is less than the maximum value.

The reduction of the scan spacing means the enlargement of the area where the laser beam overlaps, so that the energy input per unit area is proportionally increased. The

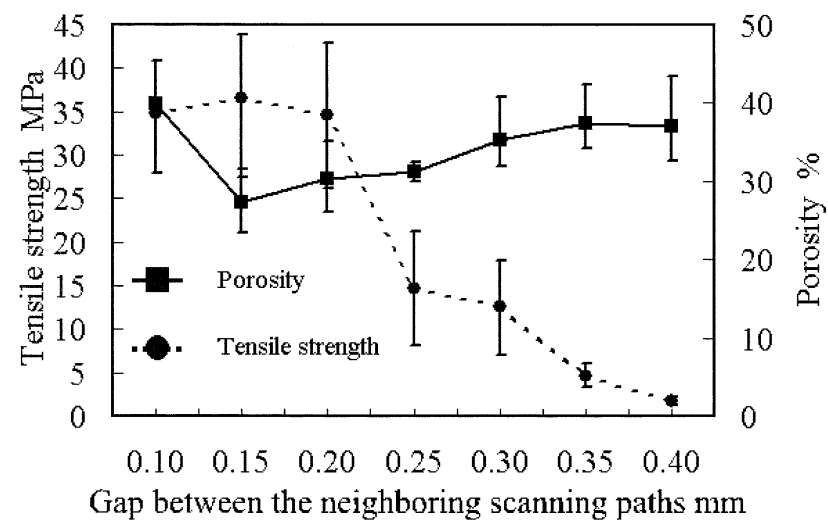

Fig. 7 Influence of scan spacing on tensile strength increase of irradiated energy causes much growth of the titanium particle size during the laser sintering process. This can be recognized in Fig. 8, which show the surface morphologies as a function of scan spacing. There exists an optimal overlap of the laser beam of about $50 \%$. When the beam spot size is $0.35 \mathrm{~mm}$, a scan spacing of $0.2 \mathrm{~mm}$ is recommended to meet the requirements of high strength and high porosity.

\subsection{Influence of average laser power on bonding strength}

In order to improve the strength of the sintered part, the influence of average power on bonding strength between neighboring layers was examined. A three-point bending test was performed in the manner shown in Fig. 5 (a): the load was applied parallel to the lamination direction. The size of the specimen and the laser scanning paths are the same as those in Fig. 4 (b), except that 6 layers of the titanium sheets were laminated by laser sintering. For laser irradiation, the average power was varied from $7.6 \mathrm{~W}$ to $25.2 \mathrm{~W}$ by increasing the pulse width from $1.7 \mathrm{~ms}$ to $3.0 \mathrm{~ms}$, but the pulse frequency of $80 \mathrm{~Hz}$, scan speed of $3.3 \mathrm{~mm} / \mathrm{s}$ and scan spacing of $0.2 \mathrm{~mm}$ were unchanged. Five specimens were prepared under each set of laser irradiation conditions for the test.

Figure 9 shows the relationship between the bending strength and the average power. Figure 10 shows the surface morphology for various average powers. The bending strength rapidly increases when the average power exceeds $20 \mathrm{~W}$. In this high-power region, it is found that plumes are generated during laser sintering. The plume

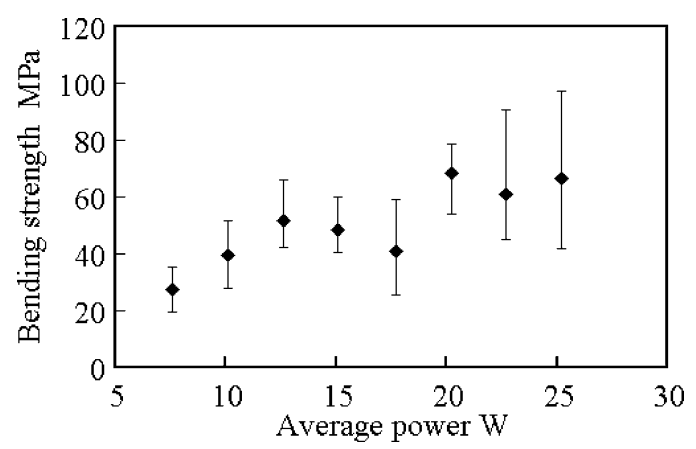

Fig. 9 Influence of average power on bending strength

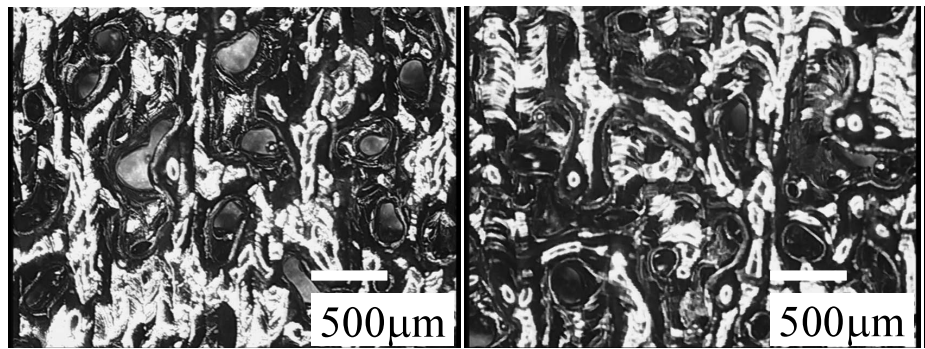

(a) $0.1 \mathrm{~mm}$ (b) $0.2 \mathrm{~mm}$

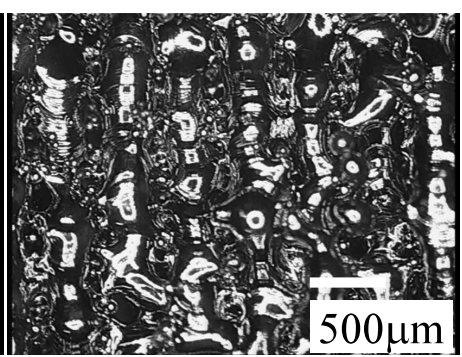

(c) $0.4 \mathrm{~mm}$

Fig. 8 Surface morphology as a function of scan spacing 


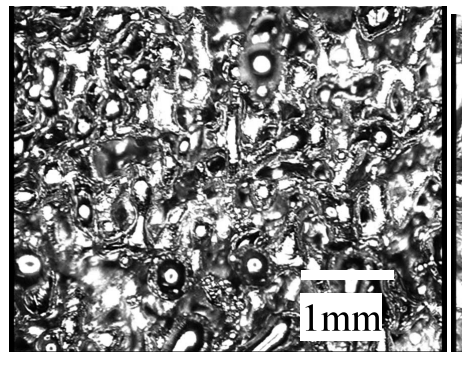

(a) $7.6 \mathrm{~W}$

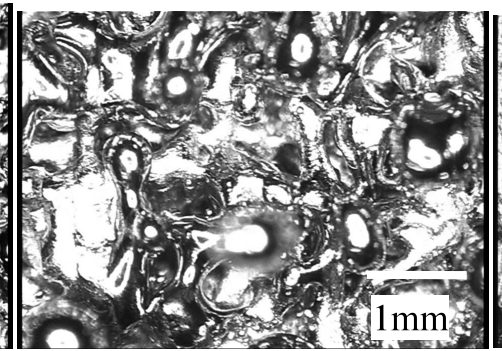

(b) $15.1 \mathrm{~W}$

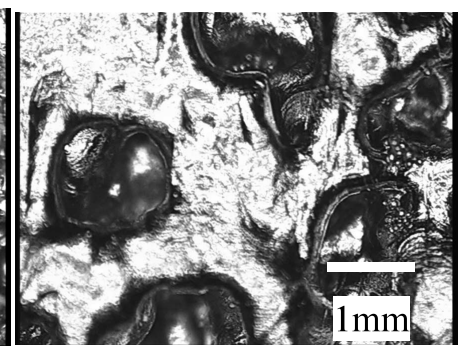

(c) $25.2 \mathrm{~W}$

Fig. 10 Surface morphology for various average powers

applies some pressure to the melt, so that neighboring layers are strongly bonded. The dispersion of the bending strength proportionally increases with average power, because an excessive laser power gives rise to rapid evaporation of the titanium particles.

The pore size becomes greater than $200 \mu \mathrm{m}$ due to surplus growth in the high-power region. A pore size of about $200 \mu \mathrm{m}$, which is suitable for osteoanagenesis, is obtained in the average power region between $12 \mathrm{~W}$ and $16 \mathrm{~W}$. In addition, Young's modulus showed the maximum value of $2.1 \mathrm{GPa}$. The strongest specimen was fabricated at an average power of $15 \mathrm{~W}$. Taking these results into consideration, we adopt, as appropriate laser irradiation conditions, an average power of $15 \mathrm{~W}$ and a pulse width of $2.22 \mathrm{~ms}$.

\section{General Discussion}

Table 2 summarizes optimum laser sintering conditions for the lamination of the titanium powder sheet when a pulsed Nd:YAG laser is used in argon atmosphere. Under these conditions, a bending strength of $63 \mathrm{MPa}$ and Young's modulus of $1.5 \mathrm{GPa}$ are attained when the load is applied parallel to the lamination direction, whereas those with load vertical to the lamination direction are $79 \mathrm{MPa}$ and $1.8 \mathrm{GPa}$, respectively. The size of pores varies from 200 to $300 \mu \mathrm{m}$, and the porosity is $63 \%$. The bending strength is almost equivalent to that of healthy human bones, ranging from $30 \mathrm{MPa}$ to $180 \mathrm{MPa}^{(8)}$. Furthermore, the strength anisotropy of $16 \mathrm{MPa}$ is within the allowable range. As for Young's modulus, the values of $1.5-$ $1.8 \mathrm{GPa}$ should be improved to around the $15 \mathrm{GPa}$ of human bones. On the other hand, the mechanical properties are expected to be improved by regenerating human bone into the porous structure of the fabricated bone after bone transplantation.

To observe the porous structures, Fig. 11 shows an SEM image of the sintered titanium part. A threedimensional network of pores can be seen. The pore size varies from 200 to $300 \mu \mathrm{m}$, and the pores are uniformly distributed over the whole matrix. These characteristics satisfy the osteoanagenesis conditions for which the suitable pore size is said to be $200-500 \mu \mathrm{m}$. The porosity
Table 2 Optimum sintering parameters of pulsed Nd:YAG laser

\begin{tabular}{l|l}
\hline Pulse frequency & $80 \mathrm{~Hz}$ \\
Pulse width & $2.22 \mathrm{~ms}$ \\
Pulse energy & $189 \mathrm{~mJ}$ \\
Peak power & $85.0 \mathrm{~W}$ \\
Average power & $15.1 \mathrm{~W}$ \\
Power density & $15.7 \mathrm{~kW} / \mathrm{cm}^{2}$ \\
Beam spot size & $350 \mu \mathrm{m}$ \\
Scan spacing & $0.2 \mathrm{~mm}$ \\
Scan speed & $3.3 \mathrm{~mm} / \mathrm{s}$ \\
\hline
\end{tabular}

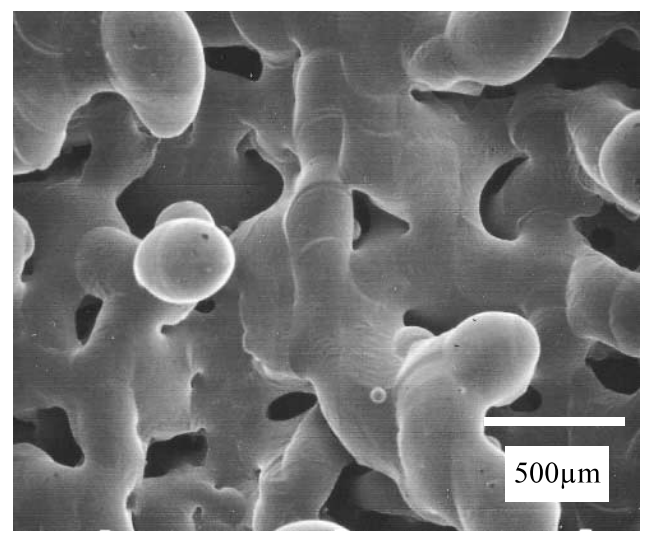

Fig. 11 SEM image of titanium artificial bone surface

measured by the Archimedean method is 63\%, which is advantageous for transplantation because that of human bones is $20-50 \%{ }^{(9)}$.

It is still required to improve the connectivity of internal porous structures since the connected neighboring pores enable the circulation of humor throughout the entire body, which aids the osteoanagenesis. It seems possible to control the internal porous structure, such as pore size and its shape, by varying the titanium particle size and shape, as well as by controlling the melting quantity within the titanium sheet in the range of $5-100 \mu \mathrm{m}$. For this purpose, the relationships between the melting depth and the laser parameters, including power density, average power, and peak power, were examined based on the laser scanning conditions listed in Table 2. The scan speed was set at $1.67 \mathrm{~mm} / \mathrm{s}, 3.33 \mathrm{~mm} / \mathrm{s}$ or $5 \mathrm{~mm} / \mathrm{s}$.

Figure 12 shows the relationship of the average power 


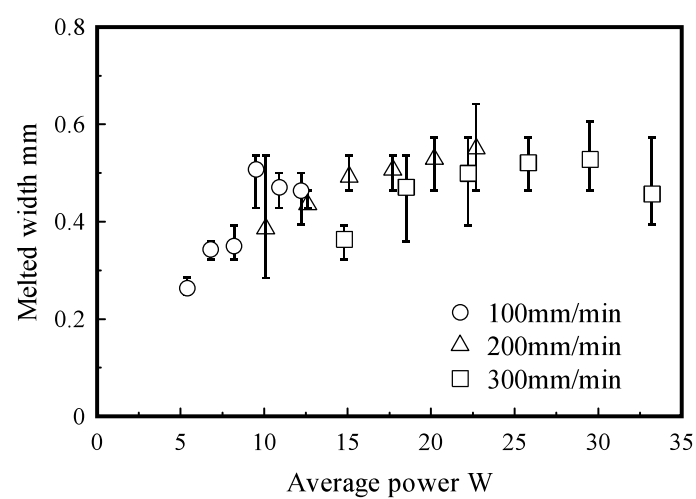

(a) Melted width

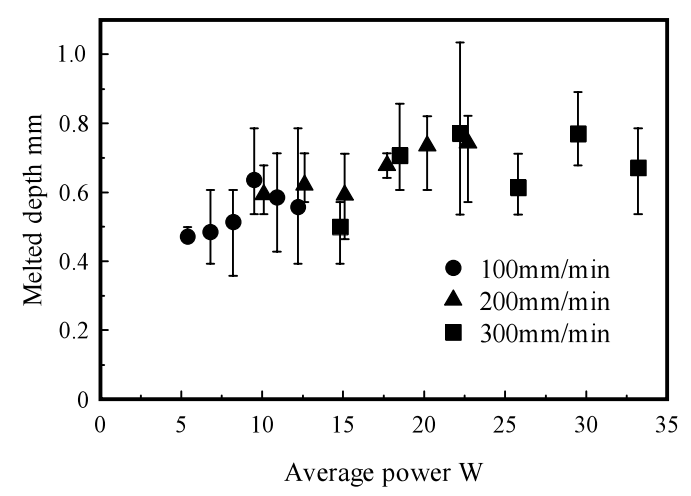

(b) Melted depth

Fig. 12 Comparison of laser melting behavior with varying average power

with (a) the melting width and (b) the melting depth of the porous titanium sheet. The pulse width was varied in the range of $2.0-4.9 \mathrm{~ms}$, and the peak power was raised from $25.6 \mathrm{~W}$ to $208 \mathrm{~W}$. Since the average power is defined as the irradiation energy per second, it increases in proportion to the pulse width, the peak power and the repetition rate, as shown in Fig. 2. Both melting width and depth uniformly increase with increasing average power in the range below $20 \mathrm{~W}$. The melting depth extends 1.2 times more than the melting width. In addition, the minimum value of both melting width and depth is $300-400 \mu \mathrm{m}$ when a beam spot diameter of $350 \mu \mathrm{m}$ and a titanium particle size of about $85 \mu \mathrm{m}$ are used. Smaller melting depth and width could be achieved by reducing both diameter sizes. Thus, melting behavior can be controlled by varying the abovementioned laser parameters.

\section{Conclusions}

A process of selective laser sintering using a titanium powder sheet has been investigated for the fabrication of porous bone substitutes. The novelty lies in the use of a sheet formed by mixing titanium powder and organic agents. The sheet is sintered layer-by-layer under argon atmosphere using a pulsed Nd:YAG laser beam. Operating parameters, such as laser irradiation and scanning paths, are extensively examined to suppress distortion, to increase strength and to adjust the pore size of the sintered part. The following major conclusions were obtained.

(1) The sintering process using short scanning paths makes it possible to reduce warpage deformation of the sintered part. This enhances adhesion between neighboring layers, leading to increases in bonding strength.

( 2 ) Under the appropriate conditions (Table 2) identified by the experiment, a bending strength of $63 \mathrm{MPa}$ and Young's modulus of $1.5 \mathrm{GPa}$ are attained when the load is applied parallel to the lamination direction, whereas those with load vertical to the lamination direction are $79 \mathrm{MPa}$ and $1.8 \mathrm{GPa}$, respectively. The size of pores varies from These values, other than Young's modulus, are almost 200 to $300 \mu \mathrm{m}$, and the porosity is approximately $65 \%$. equivalent to those of human bones.

( 3 ) Connectivity of internal porous structures must be enhanced since connected neighboring pores enable the circulation of humor throughout the entire body, which aids osteoanagenesis. The variation of the internal porous structure, such as in pore size and shape, is achieved by controlling the melting quantity in the titanium sheet within the range of about $300-400 \mu \mathrm{m}$.

\section{References}

( 1 ) Clarke, S.A., Brooks, R.A., Lee, P.T.H. and Rushton, N., The Effect of Osteogenic Growth Factors on Bone Growth into a Ceramic Filled Defect around an Implant, J. Orthop. Res., Vol.22, No.5 (2004), pp.10161024.

( 2 ) Fujibayashi, S., Neo, M., Kim, H.M., Kokubo, T. and Nakamura, T., Osteoinduction of Porous Bioactive Titanium Metal, Biomaterials, Vol.25 (2004), pp.443450.

( 3 ) Bourell, D.L., Marcus, H.L., Barlow, J.W. and Beaman, J.J., Selective Laser Sintering of Metals and Ceramics, Int. J. Powder Metallurgy, Vol.28, No.4 (1992), pp.369-381.

(4) Dietmar, W.H., Michael, S. and Makarand, V.R., Scaffold-Based Tissue Engineering: Rationale for Computer-Aided Design and Solid Free-Form Fabrication Systems, Trends in Biotechnology, Vol.22, No.7 (2004), pp.354-362.

( 5 ) Stoodley, M.A., Abbott, J.R. and Simpson, A., Titanium Cranioplasty Using 3-D Computer Modelling of Skull Defects, J. Clin. Neurosci., Vol.3, No.2 (1996), pp.149-155.

( 6 ) Abe, F., Santos, E.C., Kimura, Y., Osakada, K. and Shiomi, M., Influence of Forming Conditions on the Titanium Model in Rapid Prototyping with the Selective Laser Melting Process, J. Mech. Eng. Sci. Proc. Instn. Mech. Engrs., Vol.217, C (2003), pp.119-126.

( 7 ) Taboas, J.M., Maddox, R.D., Krebsbach, P.H. and Hollister, S.J., Indirect Solid Free Form Fabrication of Local and Global Porous, Biomimetic and Composite 3D Polymer-Ceramic Scaffolds, Biomaterials, Vol.24, Issue 1 (2003), pp.181-194.

( 8 ) Yamada, H., Strength of Biological Materials, (1970), 
p.19, Mary Land the Williams \& Wilkins Company, Baltimore Press.

( 9 ) Thieme, M., Wieters, K.P., Bergner, F., Scharnweber, D., Worch, H., Ndop, J., Kim, T.J. and Grill, W., Ti- tanium Powder Sintering for Preparation of a Porous FGM Destined as a Skeletal Replacement Implant, Mater. Sci. Forum 1999, Vol.308 (1999), p.374. 